Gut and Liver, Vol. 10, No. 6, November 2016, pp. 925-931

\title{
Performance of the Fecal Immunochemical Test for Colorectal Cancer Screening Using Different Stool-Collection Devices: Preliminary Results from a Randomized Controlled Trial
}

Hye Young Shin ${ }^{1}$, Mina Suh ${ }^{1}$, Hyung Won Baik ${ }^{1,2}$, Kui Son Choi ${ }^{1}$, Boyoung Park ${ }^{1}$, Jae Kwan Jun ${ }^{1}$, Sang-Hyun Hwang ${ }^{3}$, Byung Chang Kim ${ }^{4}$, Chan Wha Lee ${ }^{5}$, Jae Hwan Oh ${ }^{4}$, You Kyoung Lee ${ }^{6}$, Dong Soo Han ${ }^{7}$, and Do-Hoon Lee ${ }^{3}$

${ }^{1}$ National Cancer Control Institute, National Cancer Center, Goyang, ${ }^{2}$ Lung Cancer Clinic, Severance Hospital, Yonsei University Health System, Seoul, ${ }^{3}$ Department of Laboratory Medicine, Center for Diagnostic Oncology, ${ }^{4}$ Center for Colorectal Cancer, ${ }^{5}$ Center for Cancer Prevention \& Detection, National Cancer Center Hospital, National Cancer Center, Goyang, ${ }^{6}$ Department of Laboratory Medicine and Genetics, Soonchunhyang University Bucheon Hospital, Soonchunhyang University College of Medicine, Bucheon, and ${ }^{7}$ Department of Gastroenterology, Hanyang University Guri Hospital, Guri, Korea

Background/Aims: We are in the process of conducting a randomized trial to determine whether compliance with the fecal immunochemical test (FIT) for colorectal cancer screening differs according to the stool-collection method. This study was an interim analysis of the performance of two stool-collection devices (sampling bottle vs conventional container). Methods: In total, 1,701 individuals (age range, 50 to 74 years) were randomized into the sampling bottle group (intervention arm) or the conventional container group (control arm). In both groups, we evaluated the FIT positivity rate, the positive predictive value for advanced neoplasia, and the detection rate for advanced neoplasia. Results: The FIT positivity rates were $4.1 \%$ for the sampling bottles and $2.0 \%$ for the conventional containers; these values were significantly different. The positive predictive values for advanced neoplasia in the sampling bottles and conventional containers were $11.1 \%$ (95\% confidence interval [Cl], -3.4 to 25.6 ) and $12.0 \%$ ( $95 \% \mathrm{Cl},-0.7$ to 24.7 ), respectively. The detection rates for advanced neoplasia in the sampling bottles and conventional containers were 4.5 per 1,000 persons ( $95 \% \mathrm{Cl}, 2.0$ to 11.0$)$ and 2.4 per 1,000 persons ( $95 \% \mathrm{Cl}, 0.0$ to 5.0 ), respectively. Conclusions: The impact of these findings on FIT screening performance was unclear in this interim analysis. This impact should therefore be evaluated in the final analysis following the final enrollment period. (Gut Liver 2016;10:925-931)

Key Words: Colorectal neoplasms; Early detection of cancer; Screening; Intervention study; Predictive value of tests

\section{INTRODUCTION}

Population-based colorectal cancer (CRC) screening using the fecal occult blood test (FOBT) has been shown to be effective for reducing CRC mortality and incidence. ${ }^{1,2}$ Although several countries offer the FOBT in primary screening for CRC, participation has not been satisfactory. ${ }^{3}$ In Korea, the National Cancer Screening Program (NCSP) provides a fecal immunochemical test (FIT) every year to individuals who are at least 50 years old, but only $25.7 \%$ of those eligible participated in $2012 .^{4}$ To overcome poor participation, several studies evaluated the effectiveness of various strategies to improve CRC screening compliance, including CRC screening reminders by mail or telephone, provision of information on CRC screening (leaflets), and sending stool-collection devices to people's homes. ${ }^{5-7}$

To investigate strategies to increase CRC screening compliance using the FIT within the NCSP we are in the process of conducting a randomized trial using a $2 \times 2$ factorial design with the factors stool-collection device distribution method (mailing vs visiting the clinic) and type of stool-collection device (sampling bottle vs conventional container). Unlike other interventions, the type of stool-collection device can affect FIT results and CRC screening compliance. Sampling bottles (intervention arm) contain a hemoglobin ( $\mathrm{Hb}$ )-stabilizing buffer to minimize $\mathrm{Hb}$ degradation at ambient temperatures. ${ }^{8,9}$ However, the conventional containers (control arm) currently being used at the NCSP do not contain an Hb-stabilizing buffer. Therefore, we hypothesized that the sampling bottles would yield better per-

Correspondence to: Jae Kwan Jun

National Cancer Control Institute, National Cancer Center, 323 Ilsan-ro, Ilsandong-gu, Goyang 10408, Korea

Tel: +82-31-920-2184, Fax: +82-31-920-2929, E-mail: jkjun@ncc.re.kr

Received on September 22, 2015. Revised on December 24, 2015. Accepted on January 15, 2016. Published online June 13 , 2016 pISSN 1976-2283 eISSN 2005-1212 https://doi.org/10.5009/gnl15479

@) This is an Open Access article distributed under the terms of the Creative Commons Attribution Non-Commercial License (http://creativecommons.org/licenses/by-nc/4.0) which permits unrestricted non-commercial use, distribution, and reproduction in any medium, provided the original work is properly cited. 
formance than conventional containers.

In this study, we present the results of an interim analysis of the performance of the stool-collection devices. We compared FIT positivity rates (PRs) and the positive predictive values (PPVs) and detection rates (DRs) for colonic high-risk adenoma, advanced adenoma, CRC, and advanced neoplasia between the sampling bottles and the conventional containers.

\section{MATERIALS AND METHODS}

\section{Participants}

Study participants (age range, 50 to 74 years) were recruited at the National Cancer Center (NCC) and data were collected from February 2013 to January 2014. To recruit study participants, invitation letters were mailed to the target population. Details of the study protocol were published in Shin et al. ${ }^{10}$ CRC screening recipients (age range, 50 to 74 years) who were registered in the NCSP were included in the study. Participants were excluded if they had been diagnosed with any cancer, undergone colonoscopy or polypectomy, or had a FIT within the previous year. The Institutional Review Board of the NCC approved the study (NCCNCS-12-683).

\section{Study design}

This study was an interim evaluation of the performance of stool-collection devices over the course of 1 year, a duration chosen because the FIT is offered annually by the NCSP. Telephone interviews were conducted to screen for participants that met the inclusion criteria and verbal informed consent was obtained from participants before enrolment in the study. Participants were randomly allocated by a computer-generated randomization program to receive either a sampling bottle (intervention arm) or a conventional container (control arm). Participants in the intervention arm were provided with a sampling bottle (Eiken Chemical Co., Ltd., Tokyo, Japan), tissue paper to avoid contact of the stool with toilet water, and instructions for stool collection. The sampling bottle consists of a small tube with a cap. The tube contains $2.0 \mathrm{~mL} \mathrm{Hb}$-stabilizing buffer to minimize $\mathrm{Hb}$ degradation. Attached to the inside of the cap is a thin, 4.2-cm-long sampling probe used to obtain the stool sample. The sampling bottle collects approximately 10 $\mathrm{mg}$ of stool. Participants in the control arm were provided with a conventional container and instructions for stool collection. The conventional container consists of a small plastic container with a 2.0-cm-long sampling probe attached to the inside of the cap and does not contain $\mathrm{Hb}$-stabilizing buffer. All participants were instructed to collect several stool samples from one bowel movement and then to submit the samples for the FIT within 24 hours. At the NCC lab, the collected stool specimens were analyzed in an OC-SENSOR DIANA machine (Eiken Chemical Co., Ltd.), an automated analyzer designed to process the sampling bottles. Therefore, samples collected using conventional containers were transferred into sampling bottles so that they could be tested in the same machine. FIT results were mailed to participants within 3 weeks; those who received positive results were contacted by telephone and recommended for colonoscopy, as per standard medical practice. Research staff obtained informed consent from participants when they submitted their stool specimens at clinics.

\section{Outcomes and statistical analysis}

The primary outcome was the PR of the FIT based on stool samples collected using the sampling bottle or the conventional container. A positive FIT result was defined as an Hb concentration greater than $100 \mathrm{ng} / \mathrm{mL}$, based on the manufacturer's instructions, and the PR was defined as the percentage of participants with a positive FIT result among all participants. Secondary outcomes were the PPVs and DRs for high-risk adenoma, advanced adenoma, CRC, or advanced neoplasia. The PPV was defined as the percentage of participants with colonic lesions detected during a follow-up colonoscopy among participants with a positive FIT result. The DR was defined as the number of participants with colonic lesions per 1,000 participants. High-risk adenomas were defined based on the United States Multi-Society Task Force CRC guidelines for postpolypectomy surveillance. ${ }^{11}$ Briefly, adenomas were classified as high-risk if they had a villous histology, evidence of high-grade dysplasia (HGD) or were at least $10 \mathrm{~mm}$ in diameter. The presence of three or more smaller adenomas also fitted the criteria for high-risk. Adenomas were classified as advanced if they had a villous or tubulovillous pattern, evidence of HGD, or were greater than $10 \mathrm{~mm}$ in diameter. Advanced neoplasia included advanced adenoma and/or carcinoma. Expert endoscopists measured the size of colonic polyps during colonoscopy. Based on histology, polyps were classified as nonneoplastic polyp, adenoma (tubular, tubulovillous, villous, or serrate) or carcinoma by qualified pathologists.

Differences in primary and secondary outcomes between the two different stool-collection devices were analyzed using the chi-square test or Fisher exact test. Adjusted odds ratios (aORs) and 95\% confidence intervals (CIs) were calculated using multiple logistic regression analyses to compare performance between the stool-collection devices. All statistical analyses were performed using the SAS software version 9.3 (SAS Institute, Cary, NC, USA).

\section{RESULTS}

In total, 1,701 participants were included in this initial study. A study flow diagram for the enrollment process and outcomes is presented in Fig. 1. Participant characteristics are shown in Table 1. Among the participants, 1,259 and 442 submitted stool samples using the conventional container and the sampling bottle, respectively. There were no differences in general charac- 


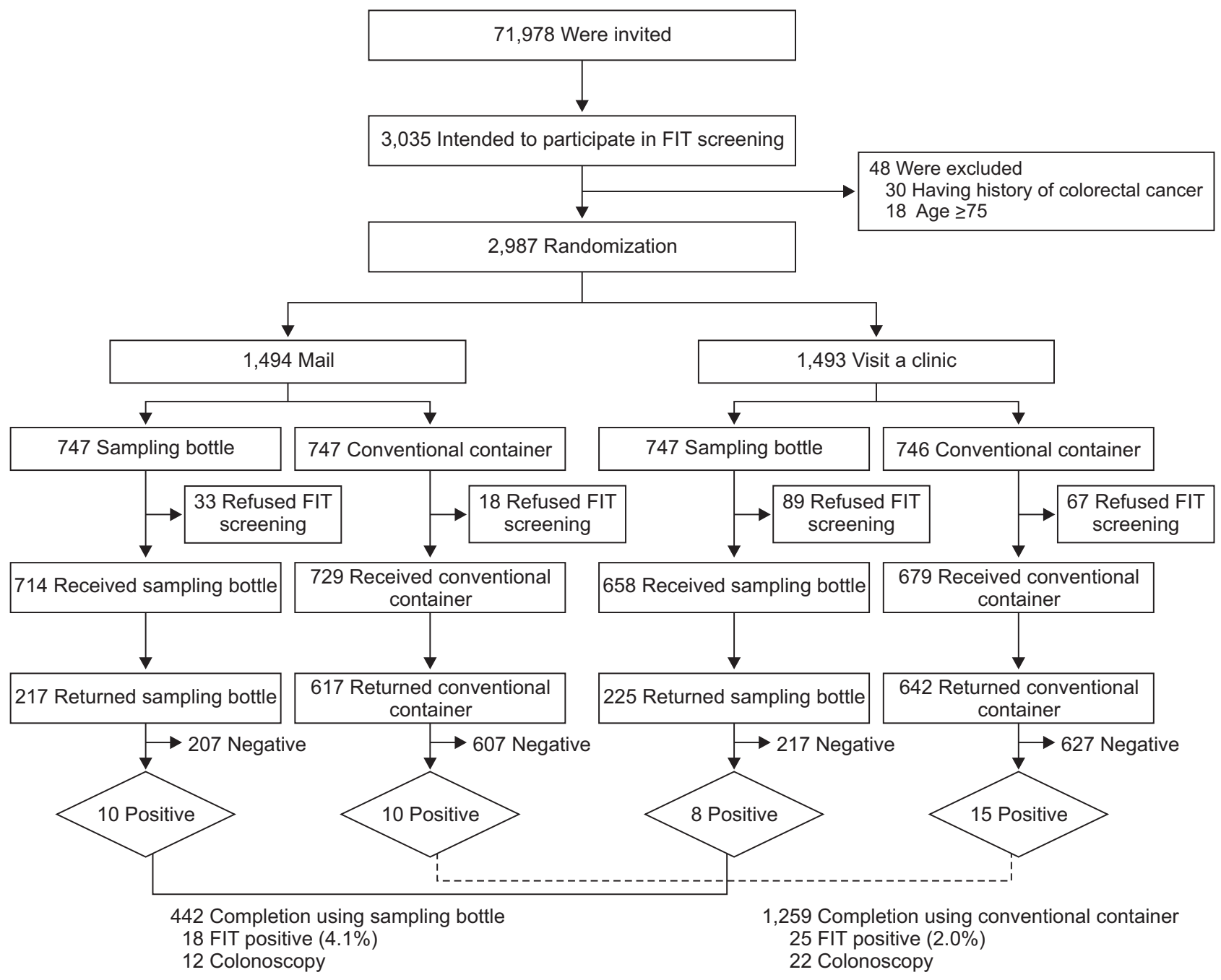

Fig. 1. Flow chart and outcomes of the study.

FIT, fecal immunochemical test.

teristics between the two groups.

Among the 43 participants who had positive FIT results, 34 underwent follow-up colonoscopy. Of the nine FIT-positive participants who did not have a colonoscopy, six were reluctant to have the procedure and three were lost to follow-up. Among those who underwent colonoscopy, 12 (66.7\%) were from the sampling bottle group and 22 (88.0\%) were from the conventional container group. For both groups, PRs, PPVs and DRs for high-risk adenoma, advanced adenoma, CRC, and advanced neoplasia are shown in Table 2. Among all participants, 43 (2.5\%) had a positive FIT result. There were $18(4.1 \%)$ and 25 participants $(2.0 \%)$ with positive FIT results in the sampling bottle and conventional container groups, respectively. The PR was significantly higher in the sampling bottle group than in the conventional container group (aOR, 1.97; 95\% CI, 1.02 to 3.79) after adjusting for sex, age, family history of CRC, medication, and smoking and alcohol consumption history. However, there were no significant differences in the PPVs or DRs for all colonic le- sions between the two groups, even high-risk adenomas and advanced neoplasias. In the sampling bottle and conventional container groups, PPVs for high-risk adenoma were 16.7\% (95\% CI, -0.5 to 33.9 ) and $12.0 \%$ (95\% CI, -0.7 to 24.7$)$, respectively. In the sampling bottle and conventional container groups, the PPVs for advanced neoplasia were $11.1 \%$ (95\% CI, -3.4 to 25.6) and $12.0 \%$ (95\% CI, -0.7 to 24.7), respectively; the DRs for highrisk adenoma were 6.8 per 1,000 persons (95\% CI, -1.0 to 14.0 ) and 2.4 per 1,000 persons ( $95 \%$ CI, 0.0 to 5.0), respectively, and the DRs for advanced neoplasia were 4.5 per 1,000 persons (95\% CI, 2.0 to 11.0 ) and 2.4 per 1,000 persons (95\% CI, 0.0 to 5.0), respectively.

\section{DISCUSSION}

The NCSP in Korea has been offering the FIT for primary CRC screening free of charge to individuals who are at least 50 years of age every year since 2004; however, CRC screening 
Table 1. Participant Characteristics Based on Stool-Collection Device

\begin{tabular}{|c|c|c|c|}
\hline \multirow{2}{*}{ Characteristic } & \multicolumn{2}{|c|}{ Type of stool-collection device } & \multirow{2}{*}{ p-value } \\
\hline & Sampling bottle & Conventional container & \\
\hline Overall & $442(100.0)$ & $1,259(100.0)$ & \\
\hline Sex & & & 0.090 \\
\hline Male & $165(37.3)$ & $528(41.9)$ & \\
\hline Female & $277(62.7)$ & $731(58.1)$ & \\
\hline Age group, yr & & & 0.218 \\
\hline $50-64$ & $324(73.3)$ & $884(70.2)$ & \\
\hline $65-74$ & $118(26.7)$ & 375 (29.8) & \\
\hline Family history of colorectal cancer & & & 0.620 \\
\hline Absent & $399(90.3)$ & $1,126(89.4)$ & \\
\hline Present & $43(9.7)$ & 133 (10.6) & \\
\hline Aspirin or NSAID or warfarin & & & 0.368 \\
\hline No & $331(74.9)$ & $942(74.8)$ & \\
\hline Yes & $81(18.3)$ & $262(20.8)$ & \\
\hline No answer & $30(6.8)$ & $55(4.4)$ & \\
\hline Smoking & & & 0.332 \\
\hline Never & $275(62.2)$ & $786(62.4)$ & \\
\hline Ever & $125(28.3)$ & $403(32.0)$ & \\
\hline No answer & $42(9.5)$ & $70(5.6)$ & \\
\hline Drinking & & & 0.617 \\
\hline Never & $220(49.8)$ & $616(48.9)$ & \\
\hline Ever & $196(44.3)$ & $581(46.2)$ & \\
\hline No answer & $26(5.9)$ & $62(4.9)$ & \\
\hline
\end{tabular}

Data are presented as number (\%).

NSAID, nonsteroidal anti-inflammatory drug.

compliance is still low compared with screening for stomach, breast, and cervical cancers. ${ }^{4}$ System-related barriers decrease participation in CRC screening; ${ }^{12}$ therefore, we are in the process of conducting a randomized trial to assess compliance according to stool-collection method. Here, we initially compared the performance of stool-collection devices (sampling bottle vs conventional container) for FIT screening.

The most interesting finding was a higher PR for sampling bottles compared with conventional containers. The PR was twice as high in the sampling bottle group (4.1\%) as in the conventional container group (2.0\%). According to the 2008 USPSTF, the PR of various commercial FIT brands was 2.0\% to 5.9\%. ${ }^{13}$ Previous studies that used a sampling bottle (OC-Sensor; Eiken Chemical Co., Ltd., Tokyo, Japan) with the same cutoff value (100 ng/mL) as our study reported similar or slightly higher PR values in the Netherlands, $4.8 \%$ to $6.0 \%{ }^{14-18}$ Italy, $4.2 \%$ and $4.5 \% ;^{19,20}$ and Belgium, 4.7\%. ${ }^{21}$ The lower PR in the conventional container group in our study was consistent with results from a Korean study that reported a PR of 2.5\% for NCSP recipients who used a conventional container for the FIT. ${ }^{22}$ The lower PR in the conventional container group may have been due to $\mathrm{Hb}$ degradation, because the conventional container does not contain $\mathrm{Hb}$-stabilizing buffer. ${ }^{8}$ Even in the presence of $\mathrm{Hb}$ stabilizing buffer, fecal $\mathrm{Hb}$ degradation can still occur ${ }^{23}$ and it has been shown in a clinical laboratory-based study that $\mathrm{Hb}$ can on average decrease by $29 \mathrm{ng} / \mathrm{mL}$ per day. ${ }^{24}$ Thus, stool samples stored in a conventional container without $\mathrm{Hb}$-stabilizing buffer are more likely to result in a negative FIT.

In a buffer-free container, a previous study found that 36.7\% of stool specimens that initially had a positive FIT result had a negative result after 24 hours at room temperature. Moreover, six positive stool specimens with $\mathrm{Hb}$ concentration of less than $200 \mathrm{ng} / \mathrm{mL}$ tested negative within 24 hours. ${ }^{9}$ In our study, all participants were instructed to submit their stool specimens to a clinic within 24 hours; however, it is possible that $\mathrm{Hb}$ degradation still occurred, resulting in the lower PR value in the conventional container. In addition, the sample probe is longer and thinner in the sampling bottle than in the conventional container and this may have made it easier for participants to sample various parts of stools, as was requested. An image of a stool-collection device is shown in Shin et al. ${ }^{10}$

The PPVs and DRs for advanced neoplasia were slightly high- 
Table 2. Performance of Sampling Bottles and Conventional Containers

\begin{tabular}{|c|c|c|c|}
\hline & \multirow{2}{*}{$\begin{array}{l}\text { Sampling bottle } \\
\qquad(\mathrm{n}=442)\end{array}$} & \multirow{2}{*}{$\begin{array}{l}\text { Conventional container } \\
\qquad(\mathrm{n}=1,259)\end{array}$} & \multirow{2}{*}{$\begin{array}{c}\text { Sampling bottle vs } \\
\text { conventional container }\end{array}$} \\
\hline & & & \\
\hline FIT positive, $\mathrm{n}(\%)$ & $18(4.1)$ & $25(2.0)$ & $1.97(1.02-3.79)$ \\
\hline Colonoscopy after positive FIT & $12(66.7)$ & $22(88.0)$ & - \\
\hline \multicolumn{4}{|l|}{ Positive predictive value, \% (95\% CI) } \\
\hline Nonadvanced adenomas & $16.7(-0.5$ to 33.9$)$ & 28.0 (10.4 to 45.6$)$ & 0.58 (0.08 to 4.07$)$ \\
\hline High-risk adenoma by USMSTF guidelines* & $16.7(-0.5$ to 33.9$)$ & $12.0(-0.7$ to 24.7$)$ & 1.33 (0.07 to 26.61) \\
\hline Advanced adenomas $^{\dagger}$ & $5.6(-5.0$ to 16.2$)$ & $4.0(-3.7$ to 11.7$)$ & - \\
\hline CRC & $5.6(-5.0$ to 16.2$)$ & $8.0(-2.6$ to 18.6$)$ & - \\
\hline Advanced neoplasia $^{\ddagger}$ & $11.1(-3.4$ to 25.6$)$ & $12.0(-0.7$ to 24.7$)$ & $3.46(0.13$ to 87.78$)$ \\
\hline \multicolumn{4}{|l|}{ Detection rate per 1,000 (95\% CI) } \\
\hline Nonadvanced adenomas & $6.8(-1.0$ to 14.0$)$ & $5.6(1.0$ to 10.0$)$ & $1.32(0.34$ to 5.19$)$ \\
\hline High-risk adenoma by USMSTF guidelines* & $6.8(-1.0$ to 14.0$)$ & $2.4(0.0$ to 5.0$)$ & 3.57 (0.70 to 18.22$)$ \\
\hline Advanced adenomas ${ }^{\dagger}$ & $2.3(-2.0$ to 7.0$)$ & $0.8(-1.0$ to 2.0$)$ & $3.82(0.23$ to 64.56$)$ \\
\hline CRC & $2.3(-2.0$ to 7.0$)$ & $1.6(-1.0$ to 4.0$)$ & $1.46(0.13$ to 16.27$)$ \\
\hline Advanced neoplasia $^{\ddagger}$ & $4.5(2.0$ to 11.0$)$ & $2.4(0.0$ to 5.0$)$ & $2.16(0.36$ to 13.10$)$ \\
\hline
\end{tabular}

The models were adjusted for sex, age, family history of colorectal cancer, medications, smoking, and alcohol consumption. aOR, adjusted odds ratio; CI, confidence interval; FIT, fecal immunochemical test; USMSTF, United States Multi-Society Task Force; CRC, colorectal cancer.

*High-risk included advanced adenoma, adenoma with villous histology, high-grade dysplasia (HGD), $\geq 3$ adenomas, or size $\geq 10$ mm; ${ }^{\dagger}$ Advanced adenoma: size $\geq 10 \mathrm{~mm}$, villous histology, or high-grade dysplasia (HGD); ${ }^{\ddagger}$ Advanced neoplasia included advanced adenoma and CRC.

er in the sampling bottle group than in the conventional container group; however, these differences were not statistically significant. Also, the values for both study groups were lower than those of previous studies using a sampling bottle (OCsensor; Eiken Chemical Co., Ltd.) with a cutoff $\mathrm{Hb}$ concentration of $100 \mathrm{ng} / \mathrm{mL}$. $^{16-18,20,25-27}$ The low values may be due to the large percentage of participants aged 50 to 64 years, whereas other studies included older subjects. ${ }^{15,16}$ Advanced neoplasias were found more frequently with increasing age. ${ }^{28}$ In addition, while the diagnostic yield was higher in males than in females, ${ }^{15,28}$ our study included a high proportion of females.

Our study has several limitations. First, the sample size was small; thus, comparison of the performance between stool-collection devices was limited. The higher PR in the sampling bottle group may result in an increase of the false positive rate, which caused subjects to undergo unnecessary colonoscopies, thus generating unnecessary costs. However, as we are still recruiting study participants, we will evaluate this performance again at the end of the study. Second, the conventional container group had a greater number of participants than that of the sampling bottle group. The data used in the present study were part of a $2 \times 2$ factorial intervention trial: (1) sampling bottle received by mail; (2) conventional container received by mail; (3) sampling bottle received at the clinic; and (4) conventional container received at the clinic. To date, the rate of compliance of those who received a sampling bottle at the clinic or via mail was lower than that in those who received the conventional container. We assume that the participants were accustomed to the use of the conventional container, because the NCSP has been offering it since 2004. Third, follow-up colonoscopy compliance after a positive FIT was lower in the sampling bottle group (66.7\%) than in the conventional container group (88.0\%) and previous studies, ${ }^{21,27}$ even though participants were encouraged (via telephone) to have a colonoscopy. Nevertheless, DRs were slightly higher in the sampling bottle group, albeit not significantly so, which suggests that a similar follow up colonoscopy compliance rate in the two groups would have facilitated comparison of the performance.

Despite these limitations, our study had several strengths. First, we used a randomized design, which minimizes bias so that the groups can be reliably compared. Second, to our knowledge, this was the first study to evaluate the performance of different stool-collection devices for CRC screening within a population-based screening program and thus we can apply the study results to the target population in the NCSP system. Additionally, our study used a cutoff $\mathrm{Hb}$ concentration of $100 \mathrm{ng} /$ $\mathrm{mL}$, which is consistent with the cutoff level of the majority of healthcare facilities that use the OC-SENSOR analyzer. Therefore, the results of this study may be applicable to the target population for CRC screening using current technological methods.

In conclusion, the PR was significantly higher in the sampling bottle group. However, the PPV and DR ware not different between the sampling bottle and conventional container groups. 
These findings may have resulted in large numbers of false positives, leading to the need for more colonoscopies and increasing costs. Therefore, the impact of our findings on the performance of FIT screening was not clear in this interim analysis; it should be further evaluated in the final analysis after the end of the final enrollment to contribute to the development of evidencebased strategies for CRC screening.

\section{CONFLICTS OF INTEREST}

No potential conflict of interest relevant to this article was reported.

\section{ACKNOWLEDGEMENTS}

This study is being supported by a Korea Health Technology R\&D Project grant through the Korea Health Industry Development Institute (KHIDI) (grant number: HI14C3411). The KHIDI is funded by the Ministry of Health \& Welfare, Republic of Korea. This study is also being supported by a grant from the National Cancer Center Foundation (NCCF) Research Project through the National Cancer Center, Republic of Korea (grant number: 1231270-1). Funding bodies have no role in the study design, study setting, analysis, or writing of the manuscript.

\section{REFERENCES}

1. Allison JE, Sakoda LC, Levin TR, et al. Screening for colorectal neoplasms with new fecal occult blood tests: update on performance characteristics. J Natl Cancer Inst 2007;99:1462-1470.

2. Jin P, Wu ZT, Li SR, et al. Colorectal cancer screening with fecal occult blood test: a 22-year cohort study. Oncol Lett 2013;6:576582.

3. Power E, Miles A, von Wagner C, Robb K, Wardle J. Uptake of colorectal cancer screening: system, provider and individual factors and strategies to improve participation. Future Oncol 2009;5:1371-1388.

4. Suh M, Choi KS, Lee YY, Jun JK. Trends in cancer screening rates among Korean men and women: results from the Korean National Cancer Screening Survey, 2004-2012. Cancer Res Treat 2013;45:86-94.

5. Green BB, Wang CY, Anderson ML, et al. An automated intervention with stepped increases in support to increase uptake of colorectal cancer screening: a randomized trial. Ann Intern Med 2013;158(5 Pt 1):301-311.

6. Ritvo PG, Myers RE, Paszat LF, et al. Personal navigation increases colorectal cancer screening uptake. Cancer Epidemiol Biomarkers Prev 2015;24:506-511.

7. Baker DW, Brown T, Buchanan DR, et al. Comparative effectiveness of a multifaceted intervention to improve adherence to annual colorectal cancer screening in community health centers: a randomized clinical trial. JAMA Intern Med 2014;174:1235-1241.
8. Vilkin A, Rozen P, Levi Z, et al. Performance characteristics and evaluation of an automated-developed and quantitative, immunochemical, fecal occult blood screening test. Am J Gastroenterol 2005;100:2519-2525.

9. Yoo SJ, Moon SJ, Gwack EH, Shin BM. Influence of the preanalytical specimen storage conditions on the fecal occult blood test results. Korean J Lab Med 2009;29:262-267.

10. Shin HY, Suh M, Baik HW, et al. The effect of changing stool collection processes on compliance in nationwide organized screening using a fecal occult blood test (FOBT) in Korea: study protocol for a randomized controlled trial. Trials 2014;15:461.

11. Lieberman DA, Rex DK, Winawer SJ, et al. Guidelines for colonoscopy surveillance after screening and polypectomy: a consensus update by the US Multi-Society Task Force on Colorectal Cancer. Gastroenterology 2012;143:844-857.

12. Park B, Choi KS, Lee YY, Jun JK, Seo HG. Cancer screening status in Korea, 2011: results from the Korean National Cancer Screening Survey. Asian Pac J Cancer Prev 2012;13:1187-1191.

13. Whitlock EP, Lin JS, Liles E, Beil TL, Fu R. Screening for colorectal cancer: a targeted, updated systematic review for the U.S. Preventive Services Task Force. Ann Intern Med 2008;149:638-658.

14. van Rossum LG, van Rijn AF, Laheij RJ, et al. Random comparison of guaiac and immunochemical fecal occult blood tests for colorectal cancer in a screening population. Gastroenterology 2008;135:82-90.

15. Hol L, Wilschut JA, van Ballegooijen M, et al. Screening for colorectal cancer: random comparison of guaiac and immunochemical faecal occult blood testing at different cut-off levels. Br J Cancer 2009;100:1103-1110.

16. Hol L, van Leerdam ME, van Ballegooijen M, et al. Screening for colorectal cancer: randomised trial comparing guaiac-based and immunochemical faecal occult blood testing and flexible sigmoidoscopy. Gut 2010;59:62-68.

17. van Roon AH, Wilschut JA, Hol L, et al. Diagnostic yield improves with collection of 2 samples in fecal immunochemical test screening without affecting attendance. Clin Gastroenterol Hepatol 2011;9:333-339.

18. de Wijkerslooth TR, Stoop EM, Bossuyt PM, et al. Immunochemical fecal occult blood testing is equally sensitive for proximal and distal advanced neoplasia. Am J Gastroenterol 2012;107:15701578.

19. Castiglione G, Grazzini G, Miccinesi G, et al. Basic variables at different positivity thresholds of a quantitative immunochemical test for faecal occult blood. J Med Screen 2002;9:99-103.

20. Grazzini G, Visioli CB, Zorzi M, et al. Immunochemical faecal occult blood test: number of samples and positivity cutoff. What is the best strategy for colorectal cancer screening? Br J Cancer 2009;100:259-265.

21. Van Roosbroeck S, Hoeck S, Van Hal G. Population-based screening for colorectal cancer using an immunochemical faecal occult blood test: a comparison of two invitation strategies. Cancer Epidemiol 2012;36:e317-e324. 
22. Park MJ, Choi KS, Lee YK, Jun JK, Lee HY. A comparison of qualitative and quantitative fecal immunochemical tests in the Korean national colorectal cancer screening program. Scand J Gastroenterol 2012;47:461-466.

23. Allison JE, Fraser CG, Halloran SP, Young GP. Population screening for colorectal cancer means getting FIT: the past, present, and future of colorectal cancer screening using the fecal immunochemical test for hemoglobin (FIT). Gut Liver 2014;8:117-130.

24. van Rossum LG, van Rijn AF, van Oijen MG, et al. False negative fecal occult blood tests due to delayed sample return in colorectal cancer screening. Int J Cancer 2009;125:746-750.

25. van Rossum LG, van Rijn AF, Laheij RJ, et al. Cutoff value determines the performance of a semi-quantitative immunochemical faecal occult blood test in a colorectal cancer screening pro- gramme. Br J Cancer 2009;101:1274-1281.

26. Brenner H, Tao S. Superior diagnostic performance of faecal immunochemical tests for haemoglobin in a head-to-head comparison with guaiac based faecal occult blood test among 2235 participants of screening colonoscopy. Eur J Cancer 2013;49:30493054.

27. Hamza S, Dancourt V, Lejeune C, Bidan JM, Lepage C, Faivre J. Diagnostic yield of a one sample immunochemical test at different cut-off values in an organised screening programme for colorectal cancer. Eur J Cancer 2013;49:2727-2733.

28. Wong MC, Lam TY, Tsoi KK, et al. A validated tool to predict colorectal neoplasia and inform screening choice for asymptomatic subjects. Gut 2014;63:1130-1136. 